\title{
Low Complexity Joint Impairment Mitigation of I/Q Modulator and PA Using Neural Networks
}

\author{
Yibo Wu, Student Member, IEEE, Ulf Gustavsson, Senior Member, IEEE, \\ Alexandre Graell i Amat, Senior Member, IEEE, and Henk Wymeersch, Senior Member, IEEE
}

\begin{abstract}
Neural networks (NNs) for multiple hardware impairments mitigation of a realistic direct conversion transmitter are impractical due to high computational complexity. We propose two methods to reduce complexity without significant performance penalty. We first propose a novel attention residual learning $\mathrm{NN}$, referred to as attention residual real-valued time-delay neural network (ARDEN), where trainable neuronwise shortcut connections between the input and output layers allow to keep the attention always active. Furthermore, we implement a NN pruning algorithm that gradually removes connections corresponding to minimal weight magnitudes in each layer. Simulation and experimental results show that ARDEN with pruning achieves better performance for compensating frequency-dependent quadrature imbalance and power amplifier nonlinearity than other NN-based and Volterra-based models, while requiring less or similar complexity.
\end{abstract}

\section{INTRODUCTION}

Radio frequency (RF) direct conversion transceivers suffer from multiple hardware impairments due to analog hardware imperfections [2] such as non-ideal digital-to-analog converters (DACs), nonlinear active lowpass filters (LPFs), imperfect local oscillators (LOs), and nonlinear power amplifiers (PAs). These impairments induce various signal distortions which degrade the quality of the transmitted signal, leading to reduced performance in terms of throughput [3]. These impairments can be mitigated separately by different algorithms, but separate optimization of each algorithm makse their combination not globally optimal.

PA nonlinearity is one of the major hardware impairments [4]. In the frequency domain, PA nonlinearity materializes as in-band errors and out-of-band emissions due to intermodulation and harmonic products [5]. PAs further exhibit memory effects during operation over large bandwidths [6], i.e., past input signals have nonlinear effects on the instantaneous output of the PA. To linearize the PA, it is customary to apply digital predistortion (DPD) [7], which compensates for the signal distortion caused by the PA nonlinearity, so that the cascade of the DPD and the PA is a linear system.

This work was presented in part at the IEEE Global Communications Conference (GLOBECOM), Taipei, Taiwan [1].

$\mathrm{Y}$. Wu is with Ericsson Research and Chalmers University of Technology, Gothenburg, Sweden (email: yibo@chalmers.se)

U. Gustavsson is with Ericsson Research, Gothenburg, Sweden (e-mail: ulf.gustavsson@ericsson.com)

A. Graell i Amat and H. Wymeersch are with Chalmers University of Technology, Gothenburg, Sweden (alexandre.graell@chalmers.se; henkw@chalmers.se)

This work was supported by the Swedish Foundation for Strategic Research (SSF), grant no. I19-0021. )
Quadrature (I/Q) imbalance is another major impairment [8], which commonly reflects as gain and phase mismatches, where the gain mismatch is introduced by the gain difference of DACs and LPFs between the in-phase (I) and quadrature (Q) branches, and the phase mismatch is caused by the LO imperfection during up- and down-conversions. Similar to the $\mathrm{PA}$, the I/Q imbalance introduces nonlinear distortions with memory effects due to the nonlinear LPFs and DACs.

Separate impairment mitigation of the PA and I/Q modulator has some shortcomings, as nonlinear mixing of the individual effects occurs. While some methods have been proposed to mitigate both impairments jointly, they suffer from either limited performance or high computational complexity [9], [10].

Several methods have been proposed to mitigate the I/Q imbalance and PA nonlinearity: Volterra series-based [8], [9], [11]-[14], NN-based [1], [10], [15]-[20], and others [21], [22]. The works [8], [21], [22] only focus on I/Q imbalance, while [11]-[13], [23] propose simplified versions of Volterra series [24] focusing only on the PA nonlinearity. Their performance is limited when both impairments occur [9]. Joint impairment mitigation of both the I/Q modulator and PA is investigated in [9], which extends the parallel Hammerstein (PH) method [11] by the finite impulse response (FIR) I/Q imbalance model so that the extended $\mathrm{PH}$ allows to jointly mitigate both I/Q modulator and PA impairments. Its performance, however, is limited for highly nonlinear PAs and I/Q modulators due to the simplification of the Volterra series and the linearity of FIR filters. All above mentioned Volterrabased models can improve performance by increasing the nonlinear order and memory length, but at the expense of an exponentially increasing complexity, which limits their utilization in practice [14].

As an alternative to Volterra-based methods, NNs for I/QPA impairments mitigation are studied in [1], [10], [15][20]. Among them, the multilayer perceptron (MLP) is mostly chosen due to easy deployment and training. Based on the MLP, the real-valued time-delay neural network (RVTDNN) was proposed by Liu et al. [15] for PA behavioral modeling. It allows to learn nonlinearities with memory effects by feeding real-valued I and $\mathrm{Q}$ components of the original complex-valued signal with time-delays. Various variants of the RVTDNN have been later proposed [1], [10], [17]-[20]. The works [1], [17], [18] only focus on the PA nonlinearity, while [10], [19] and [20] consider both frequency-flat I/Q imbalance and PA nonlinearity in single-input single-output 
(SISO) and multiple-input multiple-output (MIMO) transmitters, respectively. Specifically, our recent work [1] combines residual learning with RVTDNN, which is demonstrated to improve performance for PA nonlinearity mitigation as well as reduce complexity compared with other RVTDNN variants. A similar performance improvement is also shown in [25] for compensating nonlinearities of a fiber-optic link using residual learning NNs. None of these NN-based models consider the mitigation of nonlinear frequency-dependent I/Q imbalance, which is considerable in practice [8]. More importantly, the high-complexity problem of NN-based models is not tackled excepts in our previous work [1], which limits their usages in practice.

In this paper, we investigate the performance and complexity of impairment mitigation models for the direct conversion transceiver with multiple hardware impairments. Particularly, we consider the joint mitigation of nonlinear frequencydependent I/Q imbalance and PA nonlinearity. Our contributions are summarized as follows:

- We propose an attention residual learning $\mathrm{NN}$ based on the RVTDNN [15], referred to as attention residual realvalued time-delay neural network (ARDEN), to compensate for signal distortions caused by multiple hardware impairments, including PA nonlinearity and nonlinear frequency-dependent I/Q imbalance. Experimental results show that ARDEN yields better performance compared to state-of-the-art methods, while simultaneously exhibiting less complexity.

- We interpret the presence of an attention mechanism when learning the behavior of I/Q-PA system. We show that neurons in the first hidden layer of ARDEN fed by shorter lag input signals contribute more to the output with larger weight magnitudes, so these neurons deserve more attention.

- We propose and analyze a NN connection pruning algorithm to reduce complexity. Unimportant neural connections, i.e., those with weights with small magnitude, are gradually removed during the pruning process. Results show that pruning allows ARDEN to achieve better mitigation performance with less complexity.

- We evaluate the mitigation performance of different methods for a large complexity range. Experimental results illustrate that ARDEN with proper pruning factor performs the best over all complexity levels.

This paper extends [1] by generalizing to a multiple hardware impairments system including the PA and I/Q modulator. The weighted shortcut connections, attention mechanism, and pruning algorithm are novel.

\section{SYSTEM MOdEL}

The block diagram of a direct conversion transmitter is shown in Fig.11 The hardware impairments of the DACs, LPFs, LO, and the PA introduce I/Q imbalance and PA nonlinearity, which the DPD placed before the hardware components tris to compensate. We now describe I/Q imbalance, PA nonlinearity, and DPD in detail.

\section{A. I/Q Imbalance}

As shown in Fig.1, considering a discrete-time baseband signal $x(n)$ to be modulated by the $\mathrm{I} / \mathrm{Q}$ modulator, its real and imaginal parts, $x_{\mathrm{I}}(n)$ and $x_{\mathrm{Q}}(n)$ are sent to the $\mathrm{I}$ and $\mathrm{Q}$ branches of the modulator, respectively. We consider both wideband and frequency-dependent I/Q imbalances. The wideband I/Q imbalance is due to memoryless nonlinearities of non-ideal DACs caused by quantization noise and clipping, while the frequency-dependent I/Q imbalance is due to nonlinearities with memory effects of imperfect and non-equal LPFs. The combination of DAC and LPF is represented by the nonlinear function $f_{\mathrm{I}}: \mathbb{R}^{L_{1}+1} \rightarrow \mathbb{R}$ and $f_{\mathrm{Q}}: \mathbb{R}^{L_{1}+1} \rightarrow \mathbb{R}$ for the I and Q branches, respectively, where $L_{1}$ is the memory length. Denote the output of the DAC-LPF for the I and Q branches as $s_{\mathrm{I}}(n)$ and $s_{\mathrm{Q}}(n)$, respectively. Their input-output relations can be expressed as

$$
\begin{gathered}
s_{\mathrm{I}}(n)=f_{\mathrm{I}}\left(x_{\mathrm{I}}(n), \ldots, x_{\mathrm{I}}\left(n-L_{1}\right)\right)=f_{\mathrm{I}}\left(\boldsymbol{x}_{\mathrm{I}}^{L_{1}}\right), \\
s_{\mathrm{Q}}(n)=f_{\mathrm{Q}}\left(x_{\mathrm{Q}}(n), \ldots, x_{\mathrm{Q}}\left(n-L_{1}\right)\right)=f_{\mathrm{Q}}\left(\boldsymbol{x}_{\mathrm{Q}}^{L_{1}}\right),
\end{gathered}
$$

where $\boldsymbol{x}_{\mathrm{I}}^{L_{1}}=\left[x_{\mathrm{I}}(n), \ldots, x_{\mathrm{I}}\left(n-L_{1}\right)\right]^{\top}$, and $\boldsymbol{x}_{\mathrm{Q}}^{L_{1}}=$ $\left[x_{\mathrm{Q}}(n), \ldots, x_{\mathrm{Q}}\left(n-L_{1}\right)\right]^{\mathrm{\top}}$.

The DAC-LPF outputs are up-converted by mixers, where a phase imbalance $\phi$ is introduced, caused by LO imperfection. The output of the I/Q modulator is

$$
z(n)=z_{\mathrm{I}}(n)+\jmath z_{\mathrm{Q}}(n),
$$

where $z_{\mathrm{I}}(n)=s_{\mathrm{I}}(n)-\sin (\phi) s_{\mathrm{Q}}(n)$ and $z_{\mathrm{Q}}(n)=$ $\cos (\phi) s_{\mathrm{Q}}(n)$. Equation (3) can be rewritten as

$$
\begin{aligned}
z(n) & =s_{\mathrm{I}}(n)-\sin (\phi) s_{\mathrm{Q}}(n)+\jmath \cos (\phi) s_{\mathrm{Q}}(n) \\
& =s_{\mathrm{I}}(n)+\jmath e^{\jmath \phi} s_{\mathrm{Q}}(n) \\
& =f_{\mathrm{I}}\left(\boldsymbol{x}_{\mathrm{I}}^{L_{1}}\right)+\jmath e^{\jmath \phi} f_{\mathrm{Q}}\left(\boldsymbol{x}_{\mathrm{Q}}^{L_{1}}\right),
\end{aligned}
$$

Due to the difference between DACs and LPFs of the I and $\mathrm{Q}$ branches, $f_{\mathrm{I}}$ and $f_{\mathrm{Q}}$ present different nonlinearities and memory effects, which leads to I/Q imbalances with both frequency-independent and frequency-dependent components. For ease of notation (4), we use a single function $f_{\mathrm{IQ}}$ : $\mathbb{C}^{L_{1}+1} \rightarrow \mathbb{C}$ with memory length $L_{1}$ to represent the I/Q modulator system, so (4) can be rewritten as

$$
z(n)=f_{\mathrm{IQ}}\left(x(n), \ldots, x\left(n-L_{1}\right)\right)=f_{\mathrm{IQ}}\left(\boldsymbol{x}^{L_{1}}\right),
$$

where $\boldsymbol{x}^{L_{1}}=\left[x(n), \ldots, x\left(n-L_{1}\right)\right]^{\top}$. Note that for an ideal I/Q modulator $\phi=0, L_{1}=0$, and $z(n)=x(n)$.

\section{B. PA Nonlinearity}

The modulated signal $z(n)$ is amplified by the PA, which behaves as a nonlinear system with memory effects, i.e., the PA output at any time instant depends on the current instantaneous input and previous inputs. Memory effects are mainly due to the frequency-dependent behavior of the PA and thus more considerable for wideband signals. We define the PA as a function $f_{\mathrm{PA}}: \mathbb{C}^{L_{2}+1} \rightarrow \mathbb{C}$ with input $z(n)$ and output $y(n)$, and memory length $L_{2}$,

$$
y(n)=f_{\mathrm{PA}}\left(z(n), \ldots, z\left(n-L_{2}\right)\right)=f_{\mathrm{PA}}\left(\boldsymbol{z}^{L_{2}}\right),
$$

where $\boldsymbol{z}^{L_{2}}=\left[z(n), \ldots, z\left(n-L_{2}\right)\right]^{\top}$. For an ideal PA, $L_{2}=0$ and $y(n)=G z(n), G$ being the PA gain. 


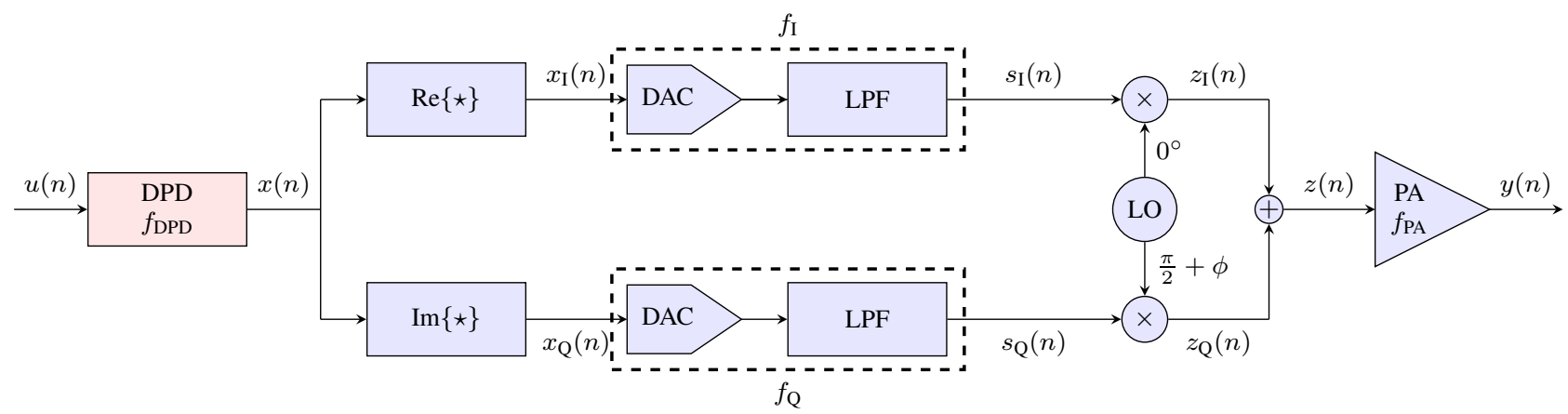

Fig. 1: Block diagram of the DPD-I/Q-PA system. The DPD block compensates for signal distortions caused by multiple hardware impairments in the direct conversion transmitter including non-ideal DACs, nonlinear LPFs, imperfect LO, and nonlinear PA.

\section{Digital Predistortion}

The DPD is represented by the function $f_{\mathrm{DPD}}: \mathbb{C}^{L_{3}+1} \rightarrow \mathbb{C}$ with memory length $L_{3}$ and input signal $u(n)$,

$$
x(n)=f_{\mathrm{DPD}}\left(u(n), \ldots, u\left(n-L_{3}\right)\right)=f_{\mathrm{DPD}}\left(\boldsymbol{u}^{L_{3}}\right),
$$

where $\boldsymbol{u}^{L_{3}}=\left[u(n), \ldots, u\left(n-L_{3}\right)\right]^{\top}$.

Substituting (5) into (6), we can rewrite $y(n)$ as

$$
\begin{aligned}
y(n) & =f_{\mathrm{PA}}\left(f_{\mathrm{IQ}}\left(\boldsymbol{x}^{L_{1}}\right), \ldots, f_{\mathrm{IQ}}\left(\boldsymbol{x}_{n-L_{2}}^{L_{1}}\right)\right) \\
& =f_{\mathrm{IQ}-\mathrm{PA}}\left(x(n), \ldots, x\left(n-L_{1}-L_{2}\right)\right) \\
& =f_{\mathrm{IQ}-\mathrm{PA}}\left(\boldsymbol{x}^{L_{1}+L_{2}}\right),
\end{aligned}
$$

where the function $f_{\mathrm{IQ}-\mathrm{PA}}: \mathbb{C}^{L_{1}+L_{2}+1} \rightarrow \mathbb{C}$ represents the I/Q-PA system. The system resulting from the cascade of the I/Q modulator and the PA has memory length $\left(L_{1}+L_{2}\right)$.

The input-output relation of the whole system is obtained by substituting (7) into (8) as

$$
\begin{aligned}
y(n) & =f_{\mathrm{IQ}-\mathrm{PA}}\left(f_{\mathrm{DPD}}\left(\boldsymbol{u}_{n}^{L_{3}}\right), \ldots, f_{\mathrm{DPD}}\left(\boldsymbol{u}_{n-L_{1}-L_{2}}^{L_{3}}\right)\right) \\
& =f_{\mathrm{DPD}-\mathrm{IQ}-\mathrm{PA}}\left(\boldsymbol{u}^{L_{1}+L_{2}+L_{3}}\right),
\end{aligned}
$$

where the function $f_{\text {DPD-IQ-PA }} \in \mathbb{C}^{L_{1}+L_{2}+L_{3}} \rightarrow \mathbb{C}$ denotes the DPD-I/Q-PA system with memory length $\left(L_{1}+L_{2}+L_{3}\right)$.

Ideally, the DPD would make the cascade DPD-I/Q-PA linear, in which case (9) would reduce to the linear function $y(n)=G u(n)$. Unfortunately, this is infeasible in practice due to the presence of hardware impairments such as PA clipping and thermal noise, which can not be compensated for. DPD methods aim, therefore, to make the DPD-I/Q-PA system as linear as possible by minimizing the mean squared error (MSE) between the PA output $y(n)$ and DPD input $u(n)$,

$$
\hat{f}_{\mathrm{DPD}}=\arg \min _{f_{\mathrm{DPD}}} \mathbb{E}\left[\left|f_{\mathrm{DPD}-\mathrm{IQ}-\mathrm{PA}}\left(\boldsymbol{u}^{L_{1}+L_{2}+L_{3}}\right)-u(n)\right|^{2}\right],
$$

where $\mathbb{E}[\cdot]$ denotes expectation.

\section{Preliminaries}

\section{A. DPD-parameter Identification by ILA}

In practice, estimating the parameters of the DPD function $f_{\text {DPD }}$ through (10) is troublesome as the I/Q-PA system is generally a combination of black boxes, i.e., unknown $f_{\text {IQ-PA }}$. The direct learning architecture (DLA) [26] solves this problem by approximating $f_{\mathrm{IQ}-\mathrm{PA}}$ as a differential model, which allows to iteratively identify DPD parameters through a gradientbased method. However, the accuracy of the identified DPD is seriously affected by the accuracy of the approximated $f_{\mathrm{IQ}-\mathrm{PA}}$, and the identification process of DLA is highly complex due to numerous updating iterations.

Instead, the indirect learning architecture (ILA) [24] indirectly estimates DPD parameters by learning the inverse behavior of the I/Q-PA system, i.e., $f_{\mathrm{IQ}-\mathrm{PA}}^{-1}$, referred to as the post-distorter, which is then used as the pre-distorter for DPD [27]. Thus, the DPD estimator (10) using ILA is changed to

$$
\hat{f}_{\mathrm{DPD}}=\arg \min _{f_{\mathrm{IO}-\mathrm{PA}}^{-1}} \mathbb{E}\left[\left|f_{\mathrm{IQ}-\mathrm{PA}}^{-1}\left(\boldsymbol{y}^{L_{1}+L_{2}}\right)-x(n)\right|^{2}\right],
$$

where $\boldsymbol{y}^{L_{1}+L_{2}}=\left[y(n), \ldots, y\left(n-L_{1}-L_{2}\right)\right]$. ILA is the most used identification method due to simple implementation and excellent performance [28]. Therefore, we consider ILA as the identification method for DPD in this paper.

\section{B. Attention Residual Learning}

The attention mechanism has been widely used in many areas such as machine translation [29], and image classification [30]. Based on the application or prior knowledge of the learning object, important features of a learning objective are highlighted by artificial attentions, such as the shape of an image or a specific word in a sentence, and those attentions help in the learning process.

Consider an unknown system $f$ with input $x$ and output $y$, i.e., $y=f(x)$. With some prior knowledge of this system, a prior estimation $f_{\text {prior }}$ of $f$ can be made, which can be helpful to further find a more accurate estimation of $f$. Thus, we say that $f_{\text {prior }}$ deserves more attention when learning $f$. This attention mechanism can be implemented by extracting $f_{\text {prior }}$ from $f$ as

$$
y=\underbrace{f_{\text {prior }}(x)}_{=f_{\text {atten }}(x)}+\underbrace{f(x)-f_{\text {prior }}(x)}_{=f_{\text {resid }}(x)},
$$

where we refer to the extracted component $f_{\text {prior }}(x)$ as the attentive function, denoted by $f_{\text {atten }}$, and the residual component $f(x)-f_{\text {prior }}(x)$ as the residual function, denoted by $f_{\text {resid }}(x)$. Thus, the prior function $f_{\text {prior }}(x)$ is now considered explicitly, i.e., being attentive, during the learning of $f(x)$, which helps 
in the learning process. In some scenarios such as image recognition, no prior knowledge of $f$ is given, so $f_{\text {prior }}(x)$ is set to $x$, which makes (12) reduce to residual learning [31]. They authors in [31] have shown that learning a residual function is more effective than learning its corresponding original function.

\section{Attention Residual LEARning NeURAL NETWORK}

In this section, we introduce the proposed ARDEN to mitigate impairments of the I/Q-PA system and NN pruning to reduce complexity.

\section{A. Attention Residual Learning for I/Q-PA System}

Since the inverse behavior of the I/Q-PA system contains the same type of hardware impairments as its forward behavior, learning the forward or backward behaviors reduces to changing the input-output and vice versa. Thus, for ease of understanding, we apply the attention mechanism described in Section III-B to analyze the forward behavior of the I/Q-PA system.

For the PA-I/Q system, the system function $f$ in (12) corresponds to (8), and the prior function $f_{\text {prior }}$ can be obtained by ignoring nonlinearities and memory effects, in which case, $f_{\mathrm{IQ}-\mathrm{PA}}$ reduces to a linear function,

$$
y(n)=W x(n)
$$

where $W$ is a mix of the linear narrowband I/Q imbalance and PA gain $G$. Following (12), we can extract the linear component (13) from the original function $(8)$ as

$$
y(n)=\underbrace{W x(n)}_{f_{\text {atten }}^{\text {I-PA }}(x(n))}+\underbrace{f_{\mathrm{IQ}-\mathrm{PA}}\left(\boldsymbol{x}^{L_{1}+L_{2}}\right)-W x(n)}_{=f_{\text {res }}^{\text {I-PA }}\left(\boldsymbol{x}^{L_{1}+L_{2}}\right)},
$$

where $f_{\text {atten }}^{\mathrm{IQ}-\mathrm{PA}}$ and $f_{\text {resid }}^{\mathrm{IQ}-\mathrm{PA}}$ denote the attentive and residual functions of the I/Q-PA system, respectively. Thus, learning the original unknown I/Q-PA behavior $f_{\mathrm{IQ}-\mathrm{PA}}$ reduces to learning the residual nonlinear behavior, as the linear behavior is always activated as attentions. Here, we refer to $W$ as the attention weight as it decides how much attention we pay to the linear input-output relation.

We remark that our attention residual learning is different from the residual learning in [31], as we only extract a specific part of the input, i.e., the current input signal $x(n)$, instead of the whole input sequence. Also, here the residual function $f_{\text {res }}^{\mathrm{IQ}-\mathrm{PA}}$ has a practical meaning that represents the nonlinear input-output relation in the I/Q-PA system, whereas the residual function in [31] does not. Furthermore, while our method is based on the attention mechanism, it also differs from the attention models in [29], [30]. Particularly, the focus of our attention is always fixed on the current input signal, i.e., the attention weights for other input signals are always zero. The selection of attention weights is based on the prior knowledge of the I/Q-PA system, as there is a strong linear relation between the input and output signals.

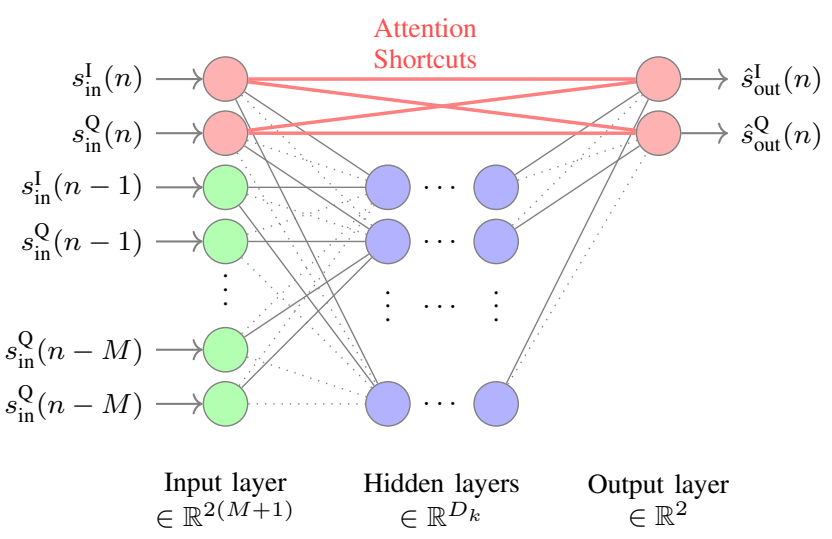

Fig. 2: Architecture of the proposed ARDEN with arbitrary connections being pruned. Dotted and solid lines between neurons represents pruned and remained connections. Fed by the real-valued I and Q components of the current and historical time instant signals, ARDEN returns estimations of the real-valued I and Q components of the current time instant output signal. When using ILA to estimate DPD parameters, $s_{\text {in }}(n)=y(n)$ and $s_{\text {out }}(n)=x(n)$.

\section{B. ARDEN Architecture}

Based on the MLP, we propose a novel NN by considering the proposed residual learning attention method (14) for the I/Q-PA system, referred to as ARDEN, and Fig.2 shows the block diagram of ARDEN with arbitrary connections pruned by the NN pruning algorithm (dotted lines), which is described in Section IV-C ARDEN consists of $K$ fully connected layers with $(K-2)$ hidden layers. The number of neurons in layer $k$ is denoted by $D_{k}$. The input vector of layer $k$ is denoted by $s_{k} \in \mathbb{R}^{D_{k-1}}$ for $k>1$. The input and output vectors of the input and output layers are $s_{1}$ and $s_{K+1}$. 11 Define a complexvalued signal with sample $s_{\text {in }}(n)=s_{\text {in }}^{\mathrm{I}}(n)+\jmath s_{\text {in }}^{\mathrm{Q}}(n)$ at time instant $n$ as the input of ARDEN. The real-valued input vector $s_{1}$ is formed by concatenating the current and previous time instants of the input signal,

$$
\boldsymbol{s}_{1}=\left[s_{\text {in }}^{\mathrm{I}}(n), s_{\text {in }}^{\mathrm{Q}}(n), \ldots, s_{\text {in }}^{\mathrm{I}}(n-M), s_{\text {in }}^{\mathrm{Q}}(n-M)\right]^{\mathrm{\top}},
$$

where $M$ denotes the number of time delays for the input signal. The time-delayed inputs allow ARDEN to capture the memory effects of the transmitter, and separating the realvalued signals allows the use of a simple real-valued training algorithm. In total, the number of neurons for the input layer is $D_{1}=(2 M+2)$.

We denote the weight matrix that connects layer $k-1$ and $k$ by $\boldsymbol{W}_{k} \in \mathbb{R}^{D_{k} \times D_{k-1}}$, for $k>1$, the $j$ th column weight vector of $\boldsymbol{W}_{k}$ by $\left[\boldsymbol{W}_{k}\right]_{j} \in \mathbb{R}^{D_{k}}$, and the corresponding bias vector by $\boldsymbol{b}_{k} \in \mathbb{R}^{D_{k}}$. For $k>1$, layers $k-1$ and $k$ are fully connected as

$$
\boldsymbol{s}_{k+1}=\sigma\left(\boldsymbol{W}_{k} \boldsymbol{s}_{k}+\boldsymbol{b}_{k}\right),
$$

where $\sigma$ denotes the element-wise activation function. To output a full range of values, the output layer is a linear layer, i.e., function $\sigma$ for the output layer is an identity mapping

\footnotetext{
${ }^{1}$ During the DPD parameter estimation using ILA, $\boldsymbol{s}_{1}$ and $\boldsymbol{s}_{K+1}$ are formed by $y(n)$ and $x(n)$, respectively, and vice versa when deploy ARDEN as DPD.
} 


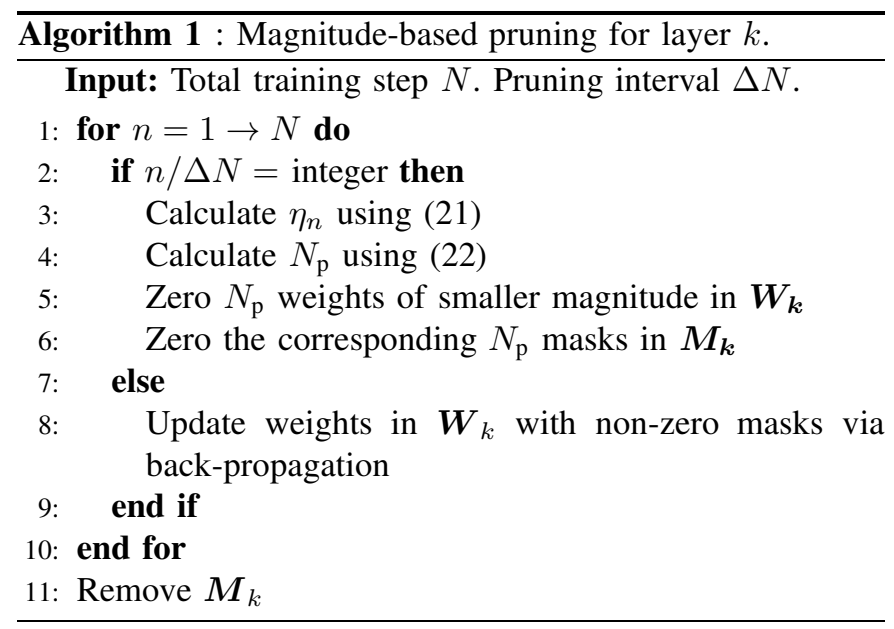

function, with number of neurons $D_{K}=2$ corresponding to the I and Q output signals.

The attentive function in (14) is implemented in ARDEN by weighted shortcut connections between the instantaneous input and output signals as

$$
f_{\text {atten }}^{\mathrm{IQ}-\mathrm{PA}}=\boldsymbol{W}_{a}\left[s_{\text {in }}^{\mathrm{I}}(n), s_{\mathrm{in}}^{\mathrm{Q}}(n)\right]^{\top},
$$

where the trainable weight matrix $\boldsymbol{W}_{a} \in \mathbb{R}^{2 \times 2}$ corresponds to a complex-valued $W$ in (13). Assuming no prior knowledge of the linear I/Q imbalance and PA gain, we initialize $\boldsymbol{W}_{a}$ as a $2 \times 2$ identity matrix. The shortcut connections are shown by red lines in Fig.2. The residual function in (14) is implemented by the hidden layers of ARDEN. Thus, the output of the output layer can be expressed as

$$
\hat{\boldsymbol{s}}_{\text {out }} \triangleq \boldsymbol{s}_{K+1}=\underbrace{\boldsymbol{W}_{a}\left[s_{\text {in }}^{\mathrm{I}}(n), s_{\text {in }}^{\mathrm{Q}}(n)\right]^{\boldsymbol{\top}}}_{f_{\text {atten }}^{\mathrm{IQ}-\mathrm{PA}}}+\underbrace{\boldsymbol{W}_{K} \boldsymbol{s}_{K}+\boldsymbol{b}_{K}}_{f_{\mathrm{res}}^{\mathrm{l} \text {-PA }}},
$$

where $\hat{\boldsymbol{s}}_{\text {out }} \in \mathbb{R}^{2}$ consists of the I and $\mathrm{Q}$ output signal estimations $\hat{s}_{\text {out }}^{\mathrm{I}}(n)$ and $\hat{s}_{\text {out }}^{\mathrm{Q}}(n)$ of the complex-valued output signal $s_{\text {out }}(n)$ at time instant $n$, respectively.

Denote all weight matrices and bias vectors as $\boldsymbol{W}=$ $\left\{\boldsymbol{W}_{1}, \ldots, \boldsymbol{W}_{K}, \boldsymbol{W}_{a}\right\}$ and $\boldsymbol{b}=\left\{\boldsymbol{b}_{1}, \ldots, \boldsymbol{b}_{K}\right\} . \boldsymbol{W}$ and $\boldsymbol{b}$ can be learned through gradient descent by minimizing the MSE between the estimation $\hat{\boldsymbol{s}}_{\text {out }}$ and observation $\boldsymbol{s}_{\text {out }}$,

$$
(\boldsymbol{W}, \boldsymbol{b})=\arg \min _{\boldsymbol{W}, \boldsymbol{b}} \mathbb{E}\left[\left|\boldsymbol{s}_{\text {out }}-\hat{\boldsymbol{s}}_{\text {out }}\right|^{2}\right] .
$$

\section{Neural Network Pruning}

NNs have been shown to achieve good performance in many tasks. However, the high computation complexity makes the deployment of NNs challenging in resource-constrained scenarios where the resource overhead for each chain, and thus for each DPD, is limited. Hence, it is crucial to reduce the complexity of NNs for DPD.

To reduce the complexity requirement, one popular technique that has been studied in recent years is NN pruning [32], [33], which reduces the NN size by removing unimportant neurons and/or connections. We apply the pruning method in [33] to reduce the complexity of ARDEN. Pruning works on each layer by adding a binary mask with the same size as the layer's weight matrix, in which a zero indicates that the weight is pruned. Let $\boldsymbol{M}_{k} \in \mathbb{R}^{D_{k} \times D_{k-1}}$ denote the binary mask matrix of layer $k$. The connection between layer $(k-1)$ and $k$ in 16 with pruning can be rewritten as

$$
\boldsymbol{s}_{k+1}=\sigma\left(\left(\boldsymbol{M}_{k} \odot \boldsymbol{W}_{k}\right) \boldsymbol{s}_{k}+\boldsymbol{b}_{k}\right),
$$

where $\odot$ denotes the Hadamard product operator. Note that shortcut connections in ARDEN are not pruned so as to keep the attention function $f_{\text {atten }}^{\text {IQ-PA }}$ always active.

Define the NN sparsity $\eta$ as the ratio of the number of zero weights to the total number of weights. Given a total number of training steps $N$, weights are pruned every $\Delta N$ steps, referred to as pruning process. Denote $\eta$ at step $n$ as $\eta_{n}$, which is gradually increased to the desired sparsity $\eta_{\mathrm{d}}$ by 33

$$
\eta_{n}=\eta_{\mathrm{d}}-\eta_{\mathrm{d}}\left(1-\frac{\lfloor n / \Delta N\rfloor}{N}\right)^{3} .
$$

The intuition behind (21) is to prune rapidly at the beginning and gradually prune less weights when the sparsity grows high. After each pruning step, non-pruned weights are retrained for $N-1$ training steps, referred to as retraining process, to alleviate the loss caused by pruning.

The pruning for layer $k$ of ARDEN is in Algorithm 1 During each pruning step, $\eta_{n}$ is calculated using (21). To meet $\eta_{n}$ for the layer with a total number of weights $N_{\mathrm{w}}$, the number of weights $N_{\mathrm{p}}$ needed to be pruned is calculated by

$$
N_{\mathrm{p}}=N_{\mathrm{w}} \times\left(1-\eta_{n}\right) .
$$

Then, weights in $\boldsymbol{W}_{k}$ are sorted by magnitudes, and the $N_{\mathrm{p}}$ weights of smaller magnitude are masked to zero by setting the corresponding values in $M_{k}$ to zero. During each retraining step, weights in $\boldsymbol{W}_{k}$ with non-zero masks are updated through $N-1$ back-propagation steps. Once pruning is done, $\boldsymbol{M}_{k}$ is removed.

\section{Computational Complexity}

We focus on the running complexity [14] of the DPD, which is defined as the number of calculations required for the inference of each output sample. Unlike the identification complexity for estimating DPD parameters that is usually done off-line, the running complexity is a real-time cost, which heavily limits the system overhead. It can be quantified by the number of multiplications and additions operated, where each real-valued multiplication or addition accounts for one floating point operations (FLOP) [14, Table. I].

We measure the complexity of ARDEN in terms of the number of FLOPs as

$$
C_{\text {arden }}=2\left(1-\eta_{\mathrm{d}}\right) \sum_{k=1}^{K-1} D_{k} D_{k+1}+8,
$$

Note that given a fixed size ARDEN, its complexity decreases linearly as $\eta_{\mathrm{d}}$ increases. where the factor 8 corresponds to the number of FLOPs introduced by the shortcut connection. Note that the required number of FLOPs decreases nearly linearly with the desired network sparsity. The computational complexity of other RVTDNN-based methods can be calculated in a similar way. 


\section{EXPERIMENTAL RESULTS}

\section{A. Setup}

1) Measurement Setup: The measurement setup is based on the RF WebLab [34], which can be remotely accessed at www.dpdcompetition.com Its block diagram is shown in Fig. 3 The block MATLAB includes all digital signal processing steps such as the DPD identification, DPD deployment, and artificial I/Q imbalance generation. In the transmission stage, digital signals generated by MATLAB are converted into analog signals by a vector signal transceiver (VST) PXIe5646R VST, and then transmitted to the Gallium Nitride PA DUT (Cree CGH4006-TB) with a $40 \mathrm{~dB}$ linear driver. In the receiving stage, through a $30 \mathrm{~dB}$ attenuator, analog $\mathrm{PA}$ output signals are collected by the VST and then sent back to MATLAB.

The baseband signal $u(n)$ used for all experiments is an orthogonal frequency division multiplexing (OFDM) signal with sampling frequency $200 \mathrm{MHz}$, signal length $10^{6}$, and bandwidth $10 \mathrm{MHz}$. An artificial I/Q imbalance is added before sending the signal to the RF WebLab. The gain imbalance is $1 \mathrm{~dB}$, and the phase imbalance is $\phi=8^{\circ}$. Frequencydependent I/Q imbalance is introduced using two 5-th order FIR lowpass elliptic filters in the I and Q branches with different filter parameters: minimum stopband attenuation of $60 \mathrm{~dB}(\mathrm{I})$ and $50 \mathrm{~dB}(\mathrm{Q})$, the peak-to-peak ripples of 0.1 $\mathrm{dB}$ (I) and $0.12 \mathrm{~dB}(\mathrm{Q})$, the normalized passband edge frequencies of $0.8(\mathrm{I})$ and $0.85(\mathrm{Q})$. For more details of the frequency response difference between these two filters, refer to [35]. The measured saturation point and measurement noise variance of the PA in RF WebLab are $24.1 \mathrm{~V}(\approx 37.6 \mathrm{dBm}$ of a $50 \Omega$ impedance) and 0.0032 , respectively. The output signal of the PA has an average power of $24.93 \mathrm{dBm}$, which corresponds to a theoretical normalized mean square error (NMSE) minimum [36] of $-39.56 \mathrm{~dB}$ and a simulated adjacent channel power ratio (ACPR) minimum [36] of $-49.92 \mathrm{dBc}$.

2) Metrics: We measure performance in terms of NMSE and ACPR. The NMSE is defined as

$$
\mathrm{NMSE}=10 \log _{10} \frac{\mathbb{E}\left[|y(n)-u(n)|^{2}\right]}{\mathbb{E}\left[|u(n)|^{2}\right]},
$$

and gives the all-band error in time-domain between the PA output signal and the DPD input signal. The ACPR is defined as

$$
\text { ACPR }=10 \log _{10} \frac{\int_{\text {adj. }}|Y(f)|^{2} \mathrm{~d} f}{\int_{\text {ch. }}|Y(f)|^{2} \mathrm{~d} f},
$$

where $Y(f)$ denotes the Fourier transform of the PA output signal. The integration in the numerator and denominator is performed over one adjacent channel (the one with larger integration between the lower and upper adjacent channel) and the main channel, respectively. The ACPR evaluates the amount of out-of-band emission.

3) Benchmarks: For a fair comparison, we consider the extended $\mathrm{PH}$ [9] because it is designed to jointly mitigate frequency-dependent I/Q imbalance and PA nonlinearity. Other referred Volterra-based models [11]-[13] fail to address both impairments. We also consider four

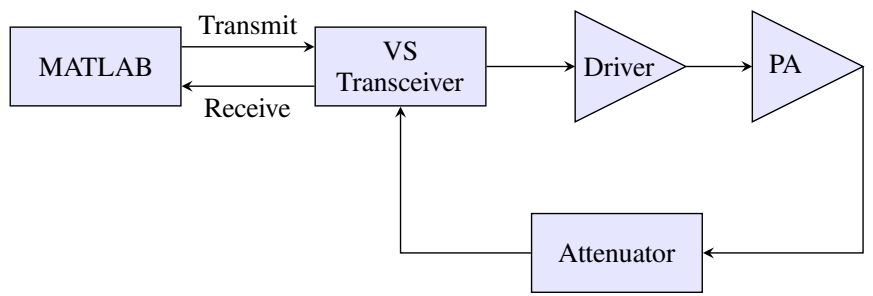

Fig. 3: Block diagram of the RF WebLab. Digital signals are transmitted and received by the block of MATLAB.

other RVTDNN-based models for comparison, namely RVTDNN [15], real-valued focused time-delay neural network (RVFTDNN) [19], augmented real-valued time-delay neural network (ARVTDNN) [10], and residual real-valued timedelay neural network (R2TDNN) [1]. All models use ILA for DPD identification. All RVTDNN-based models including the proposed ARDEN use the back-propagation algorithm with the Adam optimizer [37], the MSE loss function, the ReLU activation function, and a mini-batch size of 256 . The extended PH [9] uses the least squares algorithm for parameter identification, and its computation complexity is given in Appendix A

\section{B. Results}

1) Performance versus Complexity: Fig.4 and Fig.5 show the NMSE and ACPR as a function of the number of FLOPs for the extended PH, RVTDNN, RVFTDNN, ARVTDNN, R2TDNN [1], the proposed non-pruned ARDEN, and ARDEN with a pruning factor $\eta_{\mathrm{d}}=0.5$. For a fair comparison, all above DPD schemes have memory length 3 , i.e., $M=3$ for ARDEN. For the NN-based structures, the number of FLOPs increases as the number of neurons in each hidden layers increases. Specifically, we set the same number of hidden layers (three) for R2TDNN and ARDEN, and the same number of neurons in each hidden layer, i.e., $K=5$ and $D_{2}=D_{3}=D_{4}$. Pruned ARDEN is based on the same structure of non-pruned ARDEN. The ARVTDNN contains three augmented envelope terms of the input signal (amplitude and its square and cube) [10, Tab. II entry 11] at the input layer. For $\mathrm{PH}$, the best results are selected with respect to the number of FLOPs through an exhaustive search of different values of its nonlinear order and filter length.

The proposed ARDEN with and without pruning achieves lower NMSE and ACPR results than all other DPD schemes for all number of FLOPs. Specifically, the PH has limited mitigation performance, flattens around a NMSE of -29.9 $\mathrm{dB}$ and an ACPR of $-37.2 \mathrm{dBc}$, whereas ARDEN achieves a NMSE of $-37.0 \mathrm{~dB}$ and an ACPR of $-45.1 \mathrm{dBc}$. Compared with the R2TDNN [1], ARDEN yields sizable NMSE and ACPR gains for a number of FLOPs smaller than 500, which verifies the effectiveness of the attention weights in the shortcut connections. Furthermore, ARDEN with a pruning factor $\eta_{\mathrm{d}}=0.5$ requires even less number of FLOPs to achieve the same NMSE and ACPR compared with the nonpruned ARDEN, though this advantage vanishes as the size of ARDEN becomes large (FLOPs > 3000). 


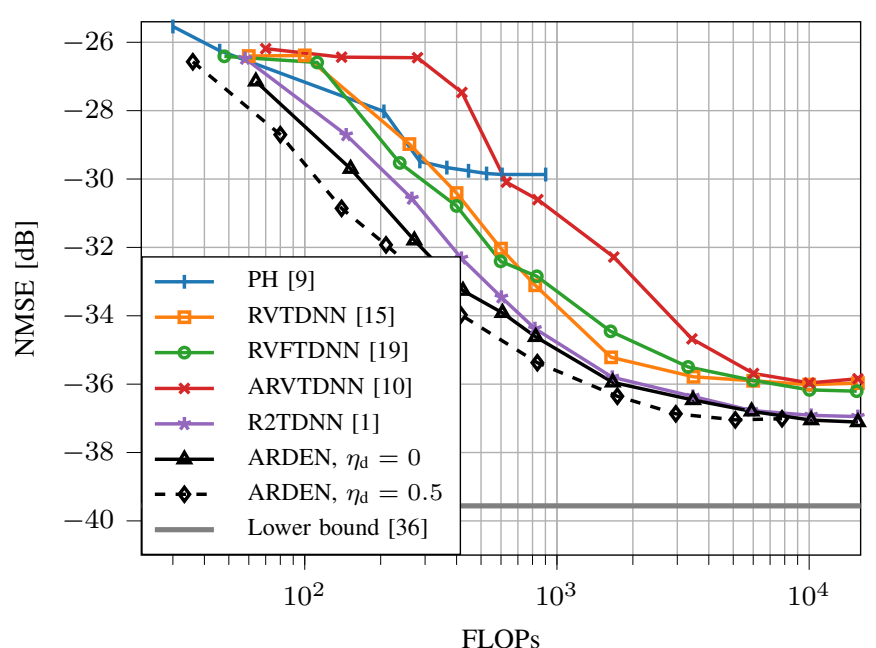

Fig. 4: NMSE as a function of the number of FLOPs for a DPD of memory length 3. The markers for $\mathrm{PH}$ [9] correspond to different sets of nonlinear order. The markers for RVTDNN [15], RVFTDNN [19], ARVTDNN [10], and ARDEN correspond to different numbers of neurons in the hidden layers. For ARDEN, $K=5$ and $D_{2}=D_{3}=D_{4}$.

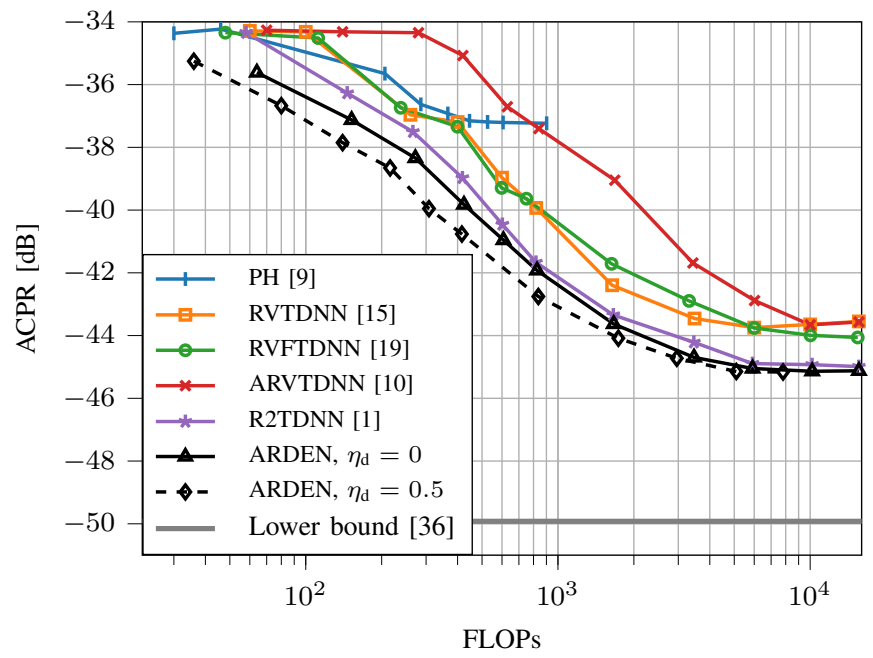

Fig. 5: ACPR as a function of the number of FLOPs for a DPD of memory length 3. The markers for $\mathrm{PH}$ [9] correspond to different sets of nonlinear order. The markers for RVTDNN [15], RVFTDNN [19], ARVTDNN [10], and ARDEN correspond to different numbers of neurons in the hidden layers. For ARDEN, $K=5$ and $D_{2}=D_{3}=D_{4}$.

2) Complexity-Restricted Scenario: We compare the mitigation performance of different DPD schemes in a limited complexity scenario for a number of FLOPs around 400. Fig.6 shows the error spectrum of the PA output without DPD, with DPD via PH, RVTDNN, RVFTDNN, ARVTDNN, ARDEN, pruned ARDEN, and of an ideal linear PA. The corresponding number of FLOPs, NMSE, and ACPR results are given in Table I The pruned ARDEN is based on an original ARDEN with $C_{\text {arden }}=818$ FLOPs and a pruning factor $\eta_{\mathrm{d}}=0.5$. For a fair comparison, the memory length for all DPD schemes is set to 3 , and the number of FLOPs for each scheme is $\approx 400$ by adjusting the number of neurons in the hidden layers for $\mathrm{NN}$-based schemes and the nonlinear order for $\mathrm{PH}$. As shown

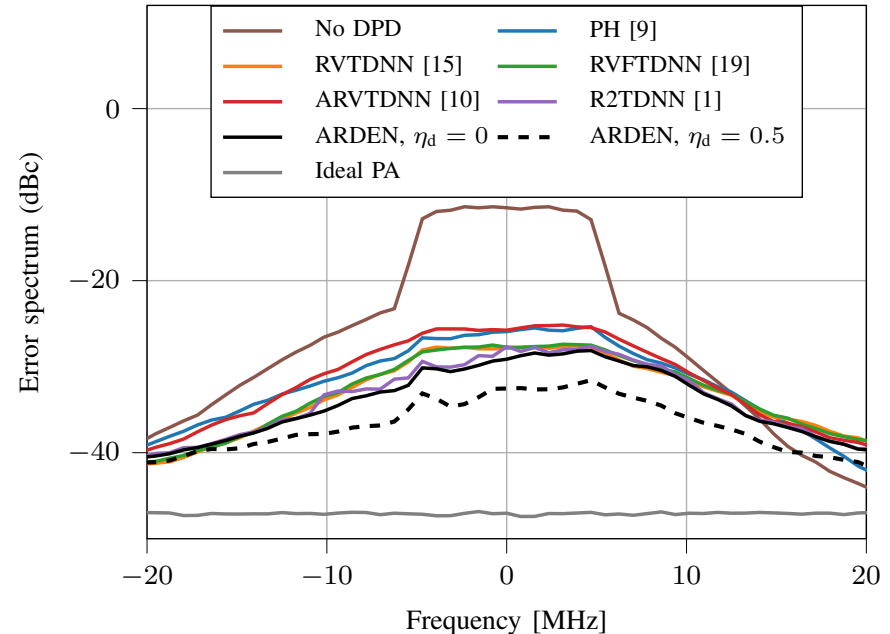

Fig. 6: Error spectrum between the actual and desired PA output signals for different models in a computation-restricted scenario with around 400 FLOPs.

TABLE I: NMSE and ACPR results of the PH [9], RVFTDNN [19 RVTDNN [15], ARVTDNN [10], and proposed ARDEN in Fig.6 The lower bound results are the minimum that can be achieved at an average output power $25.19 \mathrm{dBm}$.

\begin{tabular}{cccc}
\hline & FLOPs & NMSE [dB] & ACPR [dBc] \\
\hline No DPD & - & -17.84 & -34.40 \\
PH [9] & 446 & -29.69 & -36.76 \\
RVTDNN [15] & 400 & -31.13 & -37.91 \\
RVFTDNN [19] & 400 & -30.93 & -37.54 \\
ARVTDNN [10] & 420 & -29.22 & -36.39 \\
R2TDNN [1] & 418 & -32.06 & -38.01 \\
ARDEN, $\eta_{\mathrm{d}}=0$ & 424 & -33.26 & -38.97 \\
ARDEN, $\eta_{\mathrm{d}}=0.5$ & 416 & $-\mathbf{3 4 . 5 8}$ & $-\mathbf{4 1 . 8 2}$ \\
Lower bound [36] & - & -39.56 & -49.92 \\
\hline
\end{tabular}

in Fig6 6ithout DPD, there are considerable in-band and outof-band distortions, which are not fully compensated by any of the DPD schemes due to residual unrecoverable distortions in the I/Q-PA system. The pruned ARDEN with $\eta_{\mathrm{d}}=0.5$ achieves the best performance with NMSE of $-34.58 \mathrm{~dB}$ and ACPR of $-41.82 \mathrm{~dB}$, while requiring a similar number of FLOPs.

3) Interpretation of Pruning and Attention: Considering ARDEN with $K=3, M=3, D_{2}=512$, and $\eta_{\mathrm{d}}=0.5$, Fig.7 7 shows the cumulative distribution functions (CDFs) of the magnitude of weights in the connections between the first and second layers, i.e., $\left|\boldsymbol{W}_{2}\right|$, before and after pruning. Each CDF corresponds to the the magnitude of weights in the connections for every two neurons in the first layer fed with input signals of lag $0,1,2$, and 3, i.e., $\left|\left[\boldsymbol{W}_{2}\right]_{1,2}\right|,\left|\left[\boldsymbol{W}_{2}\right]_{3,4}\right|$, $\left|\left[\boldsymbol{W}_{2}\right]_{5,6}\right|$, and $\left|\left[\boldsymbol{W}_{2}\right]_{7,8}\right|$, respectively. The dashed and solid lines correspond to before and after pruning, respectively. The remaining weights have a minimal magnitude around 0.04 .

Note that the weights in the connections for neurons fed with shorter lag input signals have larger magnitudes $(>0.04)$ than for neurons fed with longer lag input signals, especially for lag 0 . Thus, despite the pruning factor $\eta_{\mathrm{d}}=0.5$ for the second layer, more weights $(>50 \%)$ are masked to zero for neurons with longer lags than for neurons of shorter lags 


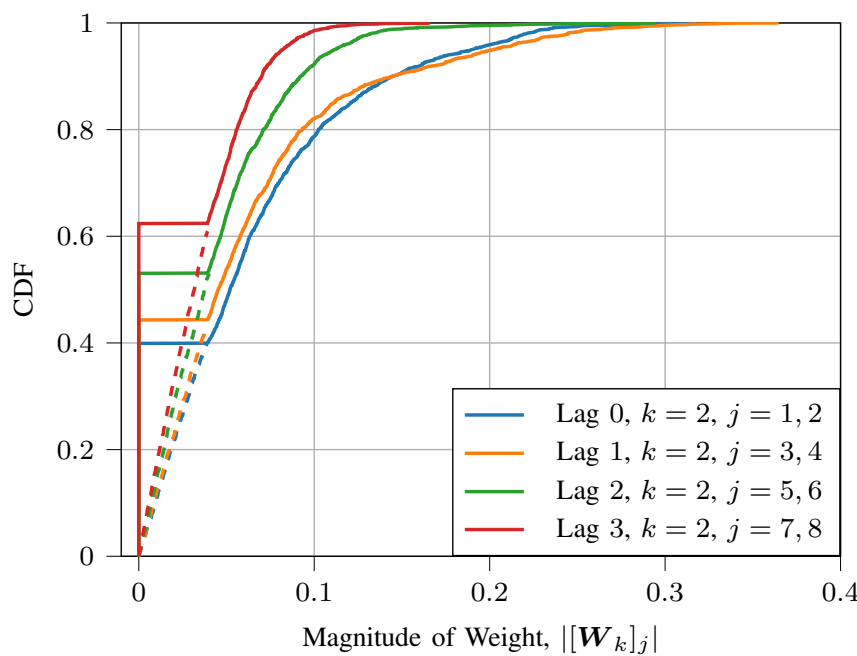

Fig. 7: CDFs of the weight magnitudes for neurons in the input layer of ARDEN fed with input signals with lag $0,1,2$, and 3 before and after pruning (dashed and solid lines), respectively. $K=3, D_{2}=512$, and $\eta_{\mathrm{d}}=0.5$. The final remaining weights have a minimal magnitude around 0.04 .

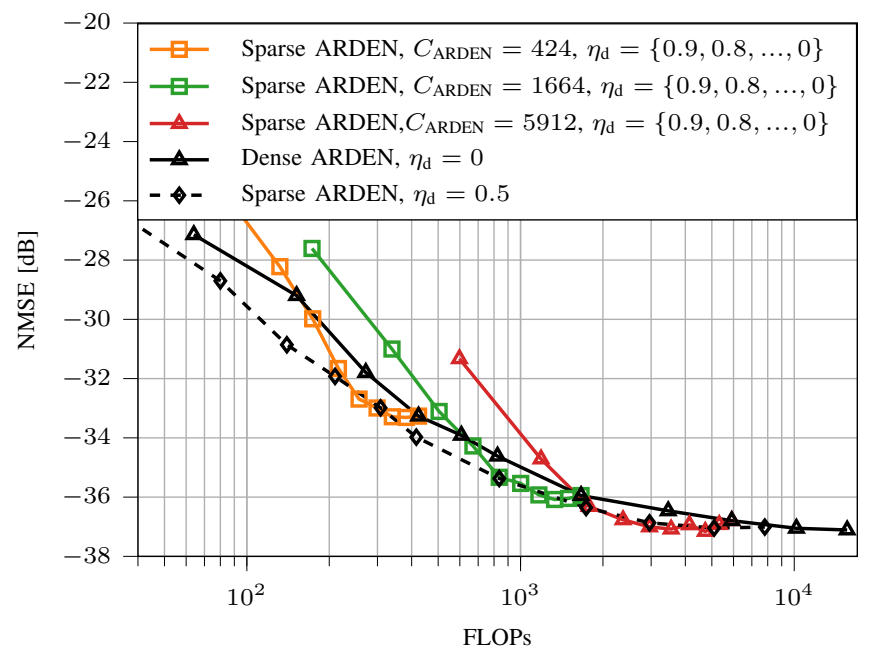

Fig. 8: NMSE as a function of the number of FLOPs for dense and sparse ARDENs with fixed $\eta_{\mathrm{d}}=\{0,0.5\}$ and varied $\eta_{\mathrm{d}}=\{0.9,0.8, \ldots, 0\}$. The markers for dense and sparse ARDENs correspond to different number of

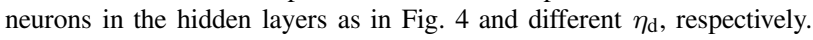

$(<50 \%)$. This indicates the presence of an inherent attention mechanism during the impairment mitigation of the I/Q-PA system.

4) Large Sparse versus Small Dense: The performance of sparse and dense ARDENs is compared in Fig.8. It illustrates the NMSE as a function of the number of FLOPs for three sparse ARDENs with a varied $\eta_{\mathrm{d}}=\{0.9,0.8, \ldots, 0\}$ but different non-pruned complexity $C_{\mathrm{ARDEN}}=\{424,1664,5912\}$, and two ARDENs with fixed $\eta_{\mathrm{d}}=\{0,0.5\}$ from Fig. 4 All the ARDENs have the same number of layers $K=5$.

For a given number of FLOPs, sparse ARDENs allow to outperform the dense ARDENs. A larger size ARDEN $\left(C_{\text {ARDEN }}=5912\right)$ allows a larger $\eta_{\mathrm{d}}=0.7$ than that of a smaller size ARDEN $\left(C_{\text {ARDEN }}=424\right)$ with $\eta_{\mathrm{d}}=0.5$. This indicates that there is an optimal pruning factor for a given sized ARDEN. The ARDEN with a fixed $\eta_{\mathrm{d}}=0.5$ performs nearly always the best over all complexities, which suggests that it is better to train a $2 \times$ larger size dense ARDEN and prune it to the desired complexity than using the best dense ARDEN.

\section{CONCLUSION}

We proposed a novel attention residual learning NN, referred to as ARDEN, for low-complexity mitigation of multiple hardware impairments in direct conversion transmitters. ARDEN keeps the instantaneous linear input-output relation of the transmitter by adding two trainable neuron-wise shortcut connections between the corresponding neurons of the input and output layers. Furthermore, we proposed and analyzed a NN connection pruning algorithm, which allows to gradually remove weights of minimum magnitude in each layer. Experimental results show that ARDEN with a pruning factor of 0.5 achieves a NMSE gain $>2.5 \mathrm{~dB}$ and an ACPR gain $>2 \mathrm{dBc}$ compared to other RVTDNN-based models and a Volterra-based model proposed in the literature, with less or similar complexity.

\section{APPENDIX A}

\section{COMPUTATION COMPLEXITY OF THE PH}

The extended PH [9] is based on the PH model [11] given by the polynomials

$$
\psi_{p}(x(n))=\sum_{k \in I_{p}} a_{k, p}|x(n)|^{k-1} x(n), p \in I_{p},
$$

where $p$ is the polynomial order, $I_{p}=\{1,3, \ldots, p\}$ for only odd orders, and $a_{k, p}$ are the polynomial weights. The polynomial (26) and its conjugate $\psi_{p}\left(x^{*}(n)\right)$ are filtered by FIR filters $h_{p}(n)$ and $h_{q}(n)$ of length $L_{p}$ and $L_{q}$, respectively. The output of the extended $\mathrm{PH}$ is

$$
y(n)=\sum_{p=1}^{P} h_{p}(n) \circledast \psi_{p}\left(x^{*}(n)\right)+\sum_{q=1}^{Q} h_{q}(n) \circledast \psi_{q}\left(x^{*}(n)\right),
$$

where $P$ and $Q$ are the polynomial orders for the nonconjugate and conjugate branches and $\circledast$ denotes convolution.

The number of complex-valued weights in (26) is

$$
N_{\mathrm{PH}, \text { poly }}=\left(1+\frac{P+1}{2}\right) \frac{P+1}{4}+\left(1+\frac{Q+1}{2}\right) \frac{Q+1}{4} .
$$

8 FLOPs are required for each weight, where 6 FLOPs are for the complex multiplication and 2 FLOPs for the complex summation [14]. Similarly, the number of complex-valued filter parameters is [9]

$$
N_{\mathrm{PH}, \text { filter }}=\sum_{p \in I_{P}} L_{p}+\sum_{q \in I_{Q}} L_{q}+1,
$$

which also require 8 FLOPs each. In total, the number of FLOPs required for the $\mathrm{PH}$ is

$C_{\mathrm{PH}}=8\left(N_{\mathrm{PH}, \text { poly }}+N_{\mathrm{PH}, \text { filter }}\right)-4+3+(\max (P, Q)-1)$. 


\section{REFERENCES}

[1] Y. Wu, U. Gustavsson, A. Graell i Amat, and H. Wymeersch, "Residual neural networks for digital predistortion," in Proc. IEEE Globecom '20, Dec. 7-11 2020, pp. 1-6.

[2] A. A. Abidi, "Direct-conversion radio transceivers for digital communications," IEEE Journal of solid-state circuits, vol. 30, no. 12, pp. 1399-1410, Dec. 1995.

[3] U. Gustavsson, C. Sanchéz-Perez, T. Eriksson, F. Athley, G. Durisi, P. Landin, K. Hausmair, C. Fager, and L. Svensson, "On the impact of hardware impairments on massive MIMO," in IEEE Globe. Workshops, 2014, pp. 294-300.

[4] S. C. Cripps, $R F$ power amplifiers for wireless communications. Artech house Norwood, MA, 2006, vol. 2.

[5] J. C. Pedro and N. B. Carvalho, Intermodulation distortion in microwave and wireless circuits. Artech House, 2003.

[6] H. Ku and J. S. Kenney, "Behavioral modeling of nonlinear RF power amplifiers considering memory effects," IEEE Trans. Microw. Theory Tech, vol. 51, no. 12, pp. 2495-2504, Dec. 2003.

[7] J. Kim and K. Konstantinou, "Digital predistortion of wideband signals based on power amplifier model with memory," Electron. Lett., vol. 37, no. 23 , pp. 1417-1418, Nov. 2001

[8] H. Cao, A. S. Tehrani, C. Fager, T. Eriksson, and H. Zirath, "I/Q imbalance compensation using a nonlinear modeling approach," IEEE Trans. Microw. Theory Tech., vol. 57, no. 3, pp. 513-518, Mar. 2009.

[9] L. Anttila, P. Händel, and M. Valkama, "Joint mitigation of power amplifier and I/Q modulator impairments in broadband direct-conversion transmitters," Trans. Microw. Theory Tech., vol. 58, no. 4, pp. 730-739, March. 2010.

[10] D. Wang, M. Aziz, M. Helaoui, and F. M. Ghannouchi, "Augmented real-valued time-delay neural network for compensation of distortions and impairments in wireless transmitters," IEEE Trans. Neural Netw. Learn. Syst, vol. 30, no. 1, pp. 242-254, Jun. 2018.

[11] L. Ding, G. T. Zhou, D. R. Morgan, Z. Ma, J. S. Kenney, J. Kim, and C. R. Giardina, "A robust digital baseband predistorter constructed using memory polynomials," IEEE Trans. Commun., vol. 52, no. 1, pp. 159-165, Jan. 2004.

[12] A. Zhu, J. C. Pedro, and T. J. Brazil, "Dynamic deviation reductionbased volterra behavioral modeling of RF power amplifiers," IEEE Trans. Microw. Theory Tech., vol. 54, no. 12, pp. 4323-4332, Dec. 2006.

[13] D. R. Morgan, Z. Ma, J. Kim, M. G. Zierdt, and J. Pastalan, "A generalized memory polynomial model for digital predistortion of RF power amplifiers," IEEE Trans. Signal Process., vol. 54, no. 10, pp. 3852-3860, Oct. 2006.

[14] A. S. Tehrani, H. Cao, S. Afsardoost, T. Eriksson, M. Isaksson, and C. Fager, "A comparative analysis of the complexity/accuracy tradeoff in power amplifier behavioral models," IEEE Trans. Microw. Theory Tech., vol. 58, no. 6, pp. 1510-1520, Jun. 2010.

[15] T. Liu, S. Boumaiza, and F. M. Ghannouchi, "Dynamic behavioral modeling of $3 \mathrm{G}$ power amplifiers using real-valued time-delay neural networks," IEEE Trans. Microw. Theory Tech., vol. 52, no. 3, pp. 10251033, Mar. 2004.

[16] M. Isaksson, D. Wisell, and D. Ronnow, "Wide-band dynamic modeling of power amplifiers using radial-basis function neural networks," IEEE Trans. Microw. Theory Tech., vol. 53, no. 11, pp. 3422-3428, Nov. 2005.

[17] M. Rawat, K. Rawat, and F. M. Ghannouchi, "Adaptive digital predistortion of wireless power amplifiers/transmitters using dynamic real-valued focused time-delay line neural networks," IEEE Trans. Microw. Theory Tech., vol. 58, no. 1, pp. 95-104, Jan. 2010.

[18] F. Mkadem and S. Boumaiza, "Physically inspired neural network model for RF power amplifier behavioral modeling and digital predistortion,"
IEEE Trans. Microw. Theory Tech., vol. 59, no. 4, pp. 913-923, Apr. 2011.

[19] M. Rawat and F. M. Ghannouchi, "A mutual distortion and impairment compensator for wideband direct-conversion transmitters using neural networks," IEEE Trans. Broadcast., vol. 58, no. 2, pp. 168-177, Jun. 2012.

[20] P. Jaraut, M. Rawat, and F. M. Ghannouchi, "Composite neural network digital predistortion model for joint mitigation of crosstalk, $I / Q$ imbalance, nonlinearity in mimo transmitters," IEEE Trans. Microw. Theory Tech., vol. 66, no. 11, pp. 5011-5020, Nov. 2018.

[21] L. Anttila, M. Valkama, and M. Renfors, "Frequency-selective I/Q mismatch calibration of wideband direct-conversion transmitters," IEEE Trans. Circuits Syst. II: Express Briefs, vol. 55, no. 4, pp. 359-363, Apr. 2008.

[22] L. Ding, Z. Ma, D. R. Morgan, M. Zierdt, and G. T. Zhou, "Compensation of frequency-dependent gain/phase imbalance in predistortion linearization systems," IEEE Trans. Circuits Syst. I: Regular Papers, vol. 55, no. 1, pp. 390-397, Feb. 2008.

[23] G. M. Raz and B. D. Van Veen, "Baseband Volterra filters for implementing carrier based nonlinearities," IEEE Trans. Signal Process., vol. 46, no. 1, pp. 103-114, Jan. 1998.

[24] C. Eun and E. J. Powers, "A new Volterra predistorter based on the indirect learning architecture," IEEE Trans. Signal Process., vol. 45, no. 1, pp. 223-227, Jan. 1997.

[25] V. Bajaj, F. Buchali, M. Chagnon, S. Wahls, and V. Aref, "Singlechannel $1.61 \mathrm{~Tb} / \mathrm{s}$ optical coherent transmission enabled by neural network-based digital pre-distortion," Proc. IEEE ECOC'20, pp. Tu1D5, Dec. 2020.

[26] D. Zhou and V. E. DeBrunner, "Novel adaptive nonlinear predistorters based on the direct learning algorithm," IEEE Trans. Signal Process., vol. 55, no. 1, pp. 120-133, Dec. 2006.

[27] M. Schetzen, "Theory of pth-order inverses of nonlinear systems," IEEE Trans. Circuits Syst., vol. 23, no. 5, pp. 285-291, May. 1976.

[28] J. Chani-Cahuana, C. Fager, and T. Eriksson, "A new variant of the indirect learning architecture for the linearization of power amplifiers," in European Microw. Conf. (EuMC), Sept. 7-10 2015, pp. 1295-1298.

[29] M.-T. Luong, H. Pham, and C. D. Manning, "Effective approaches to attention-based neural machine translation," arXiv preprint arXiv:1508.04025, 2015.

[30] F. Wang, M. Jiang, C. Qian, S. Yang, C. Li, H. Zhang, X. Wang, and X. Tang, "Residual attention network for image classification," in Proceedings of the IEEE conference on computer vision and pattern recognition, 2017, pp. 3156-3164.

[31] K. He, X. Zhang, S. Ren, and J. Sun, "Deep residual learning for image recognition," in Proc. IEEE CVPR'16, Jun. 2016, pp. 770-778.

[32] S. Han, H. Mao, and W. J. Dally, "Deep compression: Compressing deep neural networks with pruning, trained quantization and huffman coding," arXiv preprint arXiv:1510.00149, 2015.

[33] M. Zhu and S. Gupta, "To prune, or not to prune: exploring the efficacy of pruning for model compression," arXiv preprint arXiv:1710.01878, 2017.

[34] P. N. Landin, S. Gustafsson, C. Fager, and T. Eriksson, "Weblab: A webbased setup for PA digital predistortion and characterization [application notes]," IEEE Microw. Mag., vol. 16, no. 1, pp. 138-140, Feb. 2015.

[35] Z. Zhu, X. Huang, and H. Leung, "Joint I/Q mismatch and distortion compensation in direct conversion transmitters," IEEE Trans. Wireless Commun., vol. 12, no. 6, pp. 2941-2951, May. 2013.

[36] J. Chani-Cahuana, C. Fager, and T. Eriksson, "Lower bound for the normalized mean square error in power amplifier linearization," IEEE Microw. Wireless Compon. Lett., vol. 28, no. 5, pp. 425-427, May. 2018.

[37] D. P. Kingma and J. Ba, "Adam: A method for stochastic optimization," arXiv preprint arXiv:1412.6980, 2014 